\title{
Inter-Eye Correlation Analysis of 24-hour IOPs and Glaucoma Progression
}

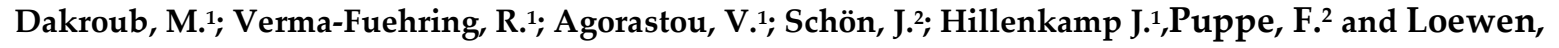 \\ N.A. ${ }^{1}$
}

1. Department of Ophthalmology, University of Würzburg, Würzburg, Germany

2. Institute for Artificial Intelligence and Knowledge Systems, Department of Informatics, University of Würzburg, Würzburg, Germany

* Correspondence: Nils Loewen, MD, PhD, Department of Ophthalmology, University Hospital Würzburg, Josef-Schneider-Straße 11, 97080 Würzburg, Germany; loewen.nils@gmail.com

\begin{abstract}
Purpose: To determine whether 24-hour IOP monitoring can be a predictor for glaucoma progression and to analyze the inter-eye relationship of IOP, perfusion and progression parameters. Methods: We extracted data from manually drawn IOP curves with HIOP-Reader, a software suite we developed. The relationship between measured IOPs and mean ocular perfusion pressures (MOPP) to retinal nerve fiber layer (RNFL) thickness was analyzed. We determined the ROC curves for peak IOP $\left(T_{\max }\right)$, average IOP(Tavg), IOP variation (IOP var) and historical IOP cut-off levels to detect glaucoma progression (rate of RNFL loss). Bivariate analysis was also conducted to check for various inter-eye relationships. Results: 217 eyes were included. The average IOP was $14.8 \pm 3.5 \mathrm{mmHg}$, with a 24 -hour variation of $5.2 \pm 2.9 \mathrm{mmHg}$. $52 \%$ of eyes with RNFL data showed disease progression. There was no significant difference in $\mathrm{T}_{\max }, \mathrm{T}_{\text {avg }}$ and IOP var between progressors and non-progressors (all $\mathrm{p}>0.05$ ). Except for $\mathrm{T}_{\text {avg }}$ and the temporal RNFL, there was no correlation between disease progression in any quadrant and $T_{\max }$, $T_{\text {avg }}$ and IOPvar. 24-hour and outpatient IOP variables had poor sensitivities and specificities in detecting disease progression. The correlation of inter-eye parameters was moderate; correlation with disease progression was weak. Conclusion: In line with our previous study, IOP data obtained during a single visit (outpatient or inpatient monitoring) make for a poor diagnostic tool, no matter the method deployed. Glaucoma progression and perfusion pressure in left and right eyes correlated weakly to moderately with each other.
\end{abstract}

Keywords: glaucoma progression; nycthemeral intraocular pressure; right-left comparison; laterality

\section{Introduction}

Open-angle glaucoma eventually becomes a bilateral disease in $50 \%$ of patients [1]. Several studies have investigated inter-eye relationships in glaucoma [2-7]. In patients with open-angle glaucoma in only one eye, chances of the contralateral eye developing this disease increase to about $40 \%$ after 2 years [7]. The probability of not just structural but functional loss in the form of a visual field defect is $25 \%$ at 5 years [2]. Visual field loss rates of both eyes are correlated in patients with bilateral glaucoma $[3,4]$ and progression in bilaterally affected eyes is faster than in patients with monocular disease [6]. Glaucomatous structure or functional deficits in one eye are predictive of contralateral eye retinal nerve fiber layer (RNFL) loss in the future [5]. One study showed that these fellow eyes had an RNFL rate loss of $1.0 \pm 0.2 \mu$ m per year, absent of other characteristics suggestive of glaucoma [5].

In several European countries, patients can be admitted for 24-h IOP monitoring to detect pressure peaks outside of regular office hours $[8,9]$ with the presumption that this is indicative of worsening or uncontrolled glaucoma. 
We recently created an extraction tool for manually charted IOP curves, HIOPReader $[9,10]$, and performed an exhaustive analysis of right eyes. We found that 24-h monitoring has limitations and, therefore, makes for a poor diagnostic tool in detecting glaucoma progression [9]. At the time, we had only included right eyes in our analysis of IOP and ocular perfusion parameters to reduce confounding variables.

The goal of this study was to examine the left eyes of the same dataset and to assess the inter-eye relationship of IOP, ocular perfusion pressure, and progression parameters. Our hypothesis was that while the use of 24-h IOP data may be flawed, these problems affect both eyes at the same time points, thereby still allowing an analysis of the inter-eye relationship that could be used to predict glaucoma. We expected IOP maxima, minima, perfusion pressures, and progression to be strongly correlated in right and left eyes.

\section{Methods}

This retrospective study was carried out at the Department of Ophthalmology at the University of Würzburg. It adhered to the core principles stated in the Declaration of Helsinki. Informed consent was waived by the Institutional Review Board of the University of Würzburg due to its retrospective nature. Two hundred twenty-five charts of patients admitted for nycthemeral (24-hour) IOP monitoring from 2017-2019 were reviewed. Diagnoses included primary open-angle glaucoma (POAG), pigmentary glaucoma (PG), juvenile glaucoma (JOAG), pseudoexfoliation glaucoma (PXG), and low-tension glaucoma (LTG). Patients with angle-closure, uveitic, neovascular glaucoma and near-complete loss of the retinal nerve fiber layer on SDOCT were excluded.

Age, diagnoses, gender, slit lamp and fundoscopic examination findings, medications, central corneal thickness, surgical history, and family history were recorded. The historical 24-hour IOP monitoring protocol in this hospital called for measurements at 10 AM, 2 PM, 5 PM, 9 PM, and 12 AM. All readings were acquired in the habitual position. The first four readings were measured in the sitting position using a Goldmann applanation tonometer (Haag-Streit, Köniz, Switzerland) while the fifth reading was acquired using a Perkins tonometer (Perkins MK3, Haag-Streit, Köniz, Switzerland) in the supine position. We wrote HIOP-Reader [9, 10], an image analysis program for high-speed extraction of data from manually drawn IOP charts. Analyzed IOP variables included $\mathrm{T}_{\text {avg, }}$ $\mathrm{T}_{\min }, \mathrm{T}_{\max }$ and IOP $\mathrm{var}\left(\mathrm{T}_{\max }-\mathrm{T}_{\min }\right)$.

\section{Image analysis of manually recorded 24-hour IOP profiles}

The evaluation was performed on standard consumer hardware from 2019 with a 2,4 GHz Quad-Core Intel Core i5-8279U CPU and 16 GB of random access memory. As described before [9], we used a Python-based program, HIOPReader [10], to extract examination date, patient name, and the IOP values on the $\mathrm{y}$-axis with their corresponding time on the x-axis. In short, we used OpenCV [11] for image processing, Tesseract [12] for optical character recognition and TensorFlow [13], and scikit-learn [14] for machine learning. We also developed a graphical user interface for the program to allow for efficient editing and error correction. The image analysis was divided into three parts: preprocessing, value detection, and name and date extraction. The main goal of preprocessing was to detect the frame containing the IOP profile and crop the image to it.

To capture the date of the 24-hour IOP profile, we applied a traditional machine learning approach. The numbers were predicted using a convolutional neural network trained on the Modified National Institute of Standards and Technology (MNIST) dataset. As the patient names were mostly recorded using machine-written labels, optical character recognition with Tesseract [16] could be used to extract all machine-written text on the form. To extract the IOP values 
entered into the profile, we detected the lines representing the different examination times using the Canny edge detection algorithm [15] and Hough line transformation [16]. We exploited the fact that all IOP values for the left eye were entered in red, while all values for the right eye were entered in blue and created color-specific masks. Since all images had the same format, the IOP value could be directly inferred from the pixel position of the detected entry.

\section{Statistical Analysis}

Data Management

Data were analyzed using SPSS Statistics (Version 26, IBM, New York, USA). Percentages were calculated for categorical variables, while means and standard deviations were computed for continuous variables. The normality of data distribution was assessed using the Kolmogorov-Smirnov test. To examine the relationship between different IOP parameters, bivariate analyses were used. For relationships between dichotomous variables (progression versus no progression) and continuous variables, a binomial logistic regression was deployed. Means were compared using the Wilcoxon signed-rank test. The correlation coefficients were interpreted using the generally considered classification of -1 being the weakest and +1 being the strongest correlation [17]. A p-value of 0.05 or less was considered statistically significant.

OCT and Glaucoma Progression Analysis

Spectral Domain OCT (SPECTRALIS OCT, Heidelberg Engineering GmbH, Heidelberg, Germany) was used to assess disease progression by measuring the retinal nerve fiber layer (RNFL) thickness (in micrometers) of the peripapillary sectors. Progression was calculated both as a dichotomous and a continuous variable using a dedicated commercial software (HEYEX Version 2.4.1., Heidelberg Engineering $\mathrm{GmbH}$, Heidelberg, Germany) by comparing the rate of RNFL loss to that of a normal age-related decline. The relationship between the slope of the RNFL loss and various continuous variables was assessed using linear regression.

\section{Results}

\section{Demographics}

In total, 217 left eyes were included in the statistical analysis. Females represented $60 \%$ of the cohort and were significantly older than males (age: 77.2 \pm 9.7 years vs $72.7 \pm 12.7$ years, for females and males, respectively, p-value $=$ 0.005). Glaucoma types included were: POAG ( $n=130,60 \%)$, PXG $(n=39,18 \%)$, LTG (n=41, $18.9 \%)$ PG (n=4, $1.8 \%)$ and JOAG (n=3, 1.4\%). Thirty-eight patients $(17.5 \%)$ were not taking any drops in their left eyes, while over a quarter $(26.8 \%)$ were taking 4 medication drops at baseline. The most common types of medication were prostaglandins $(75.6 \%)$, followed by carbonic anhydrase inhibitors $(62.2 \%)$, alpha agonists $(51.2 \%)$, and beta-blockers $(44.7 \%)$. The average central corneal thickness (CCT) was $533.4 \pm 37.7 \mu \mathrm{m}$, with no significant difference between genders $(p=0.9)$. The average MOPP was $59.5 \pm 9.3 \mathrm{mmHg}$.

\section{IOP Data Extraction}

Unlike right eyes that had their IOP values recorded in dark blue [9], left eye values were drawn into the chart using a red pencil. When using the HIOPReader on 100 IOP curves, an average of 8.4 entries per eye were recorded with a mean processing time of $3.6 \pm 0.8$ seconds per curve. The IOP data extraction was accurate, with an average of 0.5 falsely detected entries and 0.3 undetected entries per curve. The overall average IOP was $14.8 \pm 3.5 \mathrm{mmHg}$. Figure 1 shows the average IOP values measured at the five 24-hour monitoring protocol times. The means were comparable, with no significant differences between them $(\mathrm{p}=$ 0.18). $\mathrm{T}_{\max }, \mathrm{T}_{\min }$ and $\mathrm{IOP}_{\text {var }}$ were $17.4 \pm 4.4 \mathrm{mmHg}, 12.2 \pm 3.1 \mathrm{mmHg}$, and $5.2 \pm 2.9$ 
$\mathrm{mmHg}$, respectively. All 3 parameters were significantly correlated to MOPP (all p-values $<0.05$ ).

OCT Progression Data

Progression data was available in 112 out of 217 eyes. Fifty-nine eyes (52.7 $\%)$ showed a significant progression in at least one quadrant. More patients had a progression in the global peripapillary $(32.1 \%)$ and the temporal superior (32.1\%) sectors than in the temporal (23.2\%) and temporal inferior $(28.6 \%)$ sectors. Demographic parameters between progressors and non-progressors were comparable (Table 1). There were no significant differences in $\mathrm{T}_{\max }, \mathrm{T}_{\text {avg }}$ and $\mathrm{IOP}_{\text {var }}$ between progressors and non-progressors (all p-values $>0.05$ ). Except for $\mathrm{T}_{\text {avg }}$ and the temporal RNFL, there was no correlation between disease progression (rate of RNFL loss) in any quadrant and $\mathrm{T}_{\max }$, $\mathrm{T}_{\text {avg }}$ and IOP var. MOPP was found to be correlated to disease progression only in the temporal sector ( $p$ $=0.04$ ).

Table 2 shows the sensitivity and specificity of using the historical cut-off points of 15 and $22 \mathrm{mmHg}$ in detecting glaucoma progression for both 24-hour and outpatient values. These cut-off points revealed an unsatisfactory sensitivity-specificity combination in both groups. Figure 2 depicts the receiver operating characteristic (ROC) curve for the outpatient and 24-hour parameters, $\mathrm{T}_{\max }$ and $\mathrm{IOP}_{\mathrm{var}}$, in detecting disease progression. All plotted curves lie in proximity to the reference line and demonstrate the poor utility of these parameters for this purpose.

\section{Inter-Eye Analysis}

$\mathrm{T}_{\max }$ and $\mathrm{IOP}_{\mathrm{var}}$ were both significantly greater in right eyes. $\mathrm{T}_{\max }$ had a value of $19.3 \pm 5.4 \mathrm{mmHg}$ in right eyes and $17.4 \pm 4.3 \mathrm{mmHg}$ in left eyes $(\mathrm{p}<0.001)$. For $\mathrm{IOP}_{\text {var, }}$ these values were $6.9 \pm 4.2 \mathrm{mmHg}$ and $5.2 \pm 2.9 \mathrm{mmHg}$, respectively $(\mathrm{p}<$ 0.001 ). There were no significant differences between left and right eyes in the other recorded parameters ( $T_{\text {avg }}$ and $T_{\min }$ ) and in the number of disease progressors. All right IOP parameters $\left(\mathrm{T}_{\max }, \mathrm{IOP}_{\mathrm{var}}, \mathrm{T}_{\text {avg, }}\right.$ and $\left.\mathrm{T}_{\min }\right)$ showed moderate to strong positive correlations to their contralateral counterparts (Table 4). MOPP was very strongly correlated in both eyes; however, RNFL quadrants showed only a weak to moderate association (Table 4). Moreover, $74 \%$ of those who had a progression in the left eye and $67 \%$ of those who had a progression in the right eye, also had a progression in the fellow eye. Using an adjusted chi-squared test that takes inter-ocular dependency into consideration, there was a significant moderate correlation between the progression of left and right eyes $(\mathrm{p}<0.005, \mathrm{Phi}=0.32)$.

\section{Discussion}

We recently examined the utility of 24-hour (nycthemeral) IOP monitoring in detecting glaucoma progression in right eyes [9]. Here we expanded on this research and analyzed this relationship in left eyes with a focus on inter-eye correlations. IOPs from left eyes were charted using a red pencil that has a lower contrast compared to the dark blue used for right eyes analyzed in our prior study, which makes it harder to delineate by automated image recognition [18]. Despite this, IOP data from left eyes was extracted equally well by our custommade software, HIOP-Reader [9]. Once again, we found no correlation between disease progression (rate of RNFL loss) and IOP parameters extracted from 24hour monitoring, except for one between $T_{\text {avg }}$ and the temporal quadrant. This corroborates the conclusion that IOP variables, collected during a single visit, should not be used to determine the disease trajectory.

The average patient age in our study was 75 years and $60 \%$ were women, similar to what other glaucoma studies have reported $[19,20]$. Although means across at different times of the day did not differ significantly, we registered an IOP peak at $10 \mathrm{am}$ and a trough at $9 \mathrm{pm}$, similarly to our previous study. The 10 
am morning peak was delayed compared to peak IOPs reported in the literature [21-23]. The peak and trough measured here may not be accurate representations of the true peak and trough values because not enough measurements were obtained to perform a cosine fit that could have allowed to impute these values. Nevertheless, our trough was in relatively good agreement with prior studies $[21,22,24]$. The overall average IOP value was within a low IOP range because most of our patients were receiving treatment.

Surprisingly, there were no statistical differences in the baseline characteristics of progressors and non-progressors and, except for $\mathrm{T}_{\text {avg }}$ in the temporal quadrant, no IOP parameters correlated to disease progression. The fact that IOP var could not be linked to worsening glaucoma is contradictory to what has previously been reported on increased IOP fluctuations being associated with increased visual field defects in open-angle glaucoma (OAG) [25]. It is likely that too few readings and of variable quality were obtained by different on-call staff with various levels of experience. Nocturnal measurements were acquired using a Perkins handheld device, which is relatively userdependent and necessitates experience. User-independent contact lens sensors which measure IOP fluctuations constantly over 24 hours [25] or implantable sensors [26] are superior to our approach and allow IOP determination in a patient's normal environment that also captures factors such as medication adherence.

Similarly, the use of a cut-off point exceeding the individual's target IOP to predict progression did not appear to be any more useful. Our ROC curves for both left and right eyes show a poor utility of $\mathrm{T}_{\max }$ for this purpose, which highlights the importance of combining multiple parameters to effectively manage glaucoma patients. We conclude that 24-hour IOP measurements can somewhat guide therapy, but should not be used as a diagnostic tool to detect disease progression, even though IOP is a demonstrated cause of glaucoma and remains the only modifiable variable.

We found a positive correlation between IOP parameters in left and right eyes in the inter-eye correlation analysis. This is in agreement with the previously published literature that found IOP symmetry in both healthy and diseased fellow eyes [27-29]. A 2014 Chinese study on 397 healthy participants showed a very strong $(\mathrm{r}=0.83)$ interocular correlation of IOP values [27]. In treatment-naive glaucoma patients, Sit et al. demonstrated a moderate association $(r=0.54)$ of diurnal IOP patterns between fellow eyes [28]. An even stronger inter-eye correlation was found by Mansouri et al. in a similar cohort using a contact lens sensor $(r=0.76)$ [29]. However, despite the potential existence of an interocular IOP association in glaucomatous eyes, it seems to be generally weaker than in healthy eyes [30, 31]. In fact, Williams et al. identified interocular IOP asymmetry as a significant risk factor for glaucoma. An intereye asymmetry of $>6 \mathrm{mmHg}$ in IOP values was associated with a $57 \%$ probability of having glaucoma [32].

In comparison to manually obtained IOP readings, OCT measurements are far less operator dependent. Our data revealed moderate but significant correlations for RNFL rate loss between right and left eyes in all quadrants. This finding is not surprising as several studies have previously described inter-eye correlations for disease progression [2-5, 33, 34]. For instance, a study by Chen et al. in 2002 revealed an interrelationship in visual field progression rates among fellow eyes with open-angle glaucoma [4]. Interestingly, a loss of felloweye RNFL thickness has even been demonstrated in glaucoma patients showing unilateral signs of disease such as optic disc changes and visual field defects [5].

Interocular MOPP was strongly correlated in our study $(r=0.96)$. This is unsurprising, as IOP avg values did not differ significantly between both eyes, and values correlated with one another. Several studies have correlated glaucoma progression to a decrease in MOPP [35] but our study showed no significant 
association between the two. A reason for this may be the use of only one blood pressure measurement (on admission) and, thus, the inability to detect possible perfusion pressure fluctuations.

Our study had several limitations. Although the majority of glaucomas in our analysis were bilateral, some types, such as pseudoexfoliation glaucoma, are typically initially unilateral. We performed a subanalysis of PXG patients for this reason and found that the results were similar to the other patients. An important shortcoming of our study is the method of how the IOP was obtained and that patients may have had an improved medication adherence while under observation, commonly referred to as the observer or Hawthorne effect [36]. Another problem is the fact that IOP values and patterns of a single day are often not reproducible [37], an issue that can be overcome with implantable IOP sensors.

In conclusion, IOP parameters extracted from 24-hour monitoring failed to predict disease progression in left eyes. IOPs and MOPPs of left and right eyes were positively correlated with each other and the vast majority of eyes, who had a significant RNFL loss in one eye, exhibited a similar pattern in the fellow eye.

\section{Declarations:}

\section{Funding/ Support: None.}

Conflicts of Interest Statement: None of the authors have any proprietary interests or conflicts of interest related to this submission.

Data Availability: Data is available from the corresponding author on request.

Code Availability: Not applicable.

Ethics Approval: This study was approved by our Institutional Review Board (IRB), study code: 1111202 . Consent was waived by the IRB.

Consent for Participation: Not applicable.

Consent for Publication: All listed authors consent to the publication of this manuscript.

\section{References}

1. Varma R, Ying-Lai M, Francis BA, et al (2004) Prevalence of open-angle glaucoma and ocular hypertension in Latinos: the Los Angeles Latino Eye Study. Ophthalmology 111:1439-1448

2. Susanna R, Drance SM, Douglas GR (1978) The visual prognosis of the fellow eye in uniocular chronic open-angle glaucoma. Br J Ophthalmol 62:327-329

3. Anderson AJ, Gardiner SK (2020) Using the Rate of Glaucomatous Visual Field Progression in One Eye to Help Assess the Rate in the Fellow Eye. Ophthalmol Glaucoma 3:360-368

4. Chen PP (2002) Correlation of visual field progression between eyes in patients with open-angle glaucoma. Ophthalmology 109:2093-2099

5. Liu T, Tatham AJ, Gracitelli CPB, et al (2015) Rates of Retinal Nerve Fiber Layer Loss in Contralateral Eyes of Glaucoma Patients with Unilateral Progression by Conventional Methods. Ophthalmology 122:2243-2251

6. Jeong D, Sung KR, Na JH (2015) Comparison of clinical characteristics and progression rates of bilaterally and unilaterally progressing glaucoma. Korean J Ophthalmol 29:40-46

7. Nam JW, Kang YS, Sung MS, Park SW (2021) Clinical Evaluation of Unilateral Open-Angle Glaucoma: A Two-Year Follow-Up Study. Chonnam Med J 57:144-151

8. Leydhecker W (1991) Die Tagesdruckkurve. In: Leydhecker W (ed) Die Glaukome in der Praxis: Ein Leitfaden. Springer Berlin Heidelberg, Berlin, Heidelberg, pp 69-70

9. Agorastou V, Schoen J, Verma-Fuehring R, et al (2021) HIOP-reader: Automated data extraction for the analysis of manually recorded nycthemeral IOPs and glaucoma progression. Preprints

10. Schön J (2021) HIOP-Reader

11. BRADSKI, G (2000) The OpenCV library. Dr Dobb's J Software Tools 25:120-125

12. Kay A (2007) Tesseract: an open-source optical character recognition engine. Linux J 2007:2 
13. Abadi M, Agarwal A, Barham P, et al (2016) TensorFlow: Large-Scale Machine Learning on Heterogeneous Distributed Systems. arXiv [cs.DC]

14. Pedregosa F, Varoquaux G, Gramfort A, et al (2011) Scikit-learn: Machine learning in Python. the Journal of machine Learning research 12:2825-2830

15. Canny J (1986) A computational approach to edge detection. IEEE Trans Pattern Anal Mach Intell 8:679_ 698

16. Hough PVC Method and means for recognizing complex patterns. 1962. US patent

17. Taylor R (1990) Interpretation of the Correlation Coefficient: A Basic Review. J Diagn Med Sonogr 6:3539

18. Miao H, Guo X, Chen Y (2014) Application of automatic threshold in dynamic target recognition with low contrast. International Symposium on Optoelectronic Technology and Application 2014: Image Processing and Pattern Recognition

19. Quigley HA, Broman AT (2006) The number of people with glaucoma worldwide in 2010 and 2020 . Br J Ophthalmol 90:262-267

20. National Center for Health Statistics (US) (2021) Health, United States, 2019. National Center for Health Statistics (US), Hyattsville (MD)

21. Liu JHK, Zhang X, Kripke DF, Weinreb RN (2003) Twenty-four-hour intraocular pressure pattern associated with early glaucomatous changes. Invest Ophthalmol Vis Sci 44:1586-1590

22. Lee YR, Kook MS, Joe SG, et al (2012) Circadian (24-hour) pattern of intraocular pressure and visual field damage in eyes with normal-tension glaucoma. Invest Ophthalmol Vis Sci 53:881-887

23. Saccà SC, Rolando M, Marletta A, et al (1998) Fluctuations of intraocular pressure during the day in openangle glaucoma, normal-tension glaucoma and normal subjects. Ophthalmologica 212:115-119

24. David R, Zangwill L, Briscoe D, et al (1992) Diurnal intraocular pressure variations: an analysis of 690 diurnal curves. Br J Ophthalmol 76:280-283

25. Tojo N, Hayashi A, Otsuka M (2020) Correlation between 24-h continuous intraocular pressure measurement with a contact lens sensor and visual field progression. Graefes Arch Clin Exp Ophthalmol 258:175-182

26. Mansouri K, Rao HL, Weinreb RN, ARGOS-02 Study Group (2020) Short-Term and Long-Term Variability of Intraocular Pressure Measured with an Intraocular Telemetry Sensor in Patients with Glaucoma. Ophthalmology 128:227-233

27. Li Y, Bao FJ (2014) Interocular symmetry analysis of bilateral eyes. J Med Eng Technol 38:179-187

28. Sit AJ, Liu JHK, Weinreb RN (2006) Asymmetry of right versus left intraocular pressures over 24 hours in glaucoma patients. Ophthalmology 113:425-430

29. Mansouri K, Gillmann K (2020) Intereye Symmetry of 24-Hour Intraocular Pressure-related Patterns in Untreated Glaucoma Patients Using a Contact Lens Sensor. J Glaucoma 29:666

30. Liu JHK, Weinreb RN (2014) Asymmetry of habitual 24-hour intraocular pressure rhythm in glaucoma patients. Invest Ophthalmol Vis Sci 55:7398-7402

31. Liu JHK, Sit AJ, Weinreb RN (2005) Variation of 24-hour intraocular pressure in healthy individuals: right eye versus left eye. Ophthalmology 112:1670-1675

32. Williams AL, Gatla S, Leiby BE, et al (2013) The value of intraocular pressure asymmetry in diagnosing glaucoma. J Glaucoma 22:215-218

33. Teng B, Li D, Choi EY, et al (2020) Inter-Eye Association of Visual Field Defects in Glaucoma and Its Clinical Utility. Transl Vis Sci Technol 9:22

34. Fontana L, Armas R, Garway-Heath DF, et al (1999) Clinical factors influencing the visual prognosis of the fellow eyes of normal tension glaucoma patients with unilateral field loss. British Journal of Ophthalmology 83:1002-1005

35. Raman P, Suliman NB, Zahari M, et al (2018) Low nocturnal diastolic ocular perfusion pressure as a risk factor for NTG progression: a 5-year prospective study. Eye 32:1183-1189

36. McCarney R, Warner J, Iliffe S, et al (2007) The Hawthorne Effect: a randomised, controlled trial. BMC Med Res Methodol 7:30

37. Realini T, Weinreb RN, Wisniewski S (2011) Short-term repeatability of diurnal intraocular pressure patterns in glaucomatous individuals. Ophthalmology 118:47-51 


\section{Tables}

Table 1 Demographics

Table 2 Comparison of the demographic parameters of progressors and non-progressors in left eyes Table 3 Utility of cut-off IOP values with sensitivity and specificity determined for cut-off points 15 and $22 \mathrm{mmHg}$ for detecting glaucoma progression in left eyes. OP-IOP = IOP measurements during outpatient hours (10 AM, 2 PM, 5 PM)

Table 4 Bivariate correlations of various measured parameters between left and right eyes. All correlations had a p-value $<0.05$. RNFL = Slope of Retinal Nerve Fiber Layer Thickness decrease; G $=$ Global; $\mathrm{TS}=$ temporal-superior; $\mathrm{T}=$ temporal; $\mathrm{TI}=$ temporal-inferior

\section{Figures}

Fig. 1 Average IOP values measured at the 5 nycthemeral monitoring protocol times. The peak IOP was recorded at 10 am and the trough at $9 \mathrm{pm}$

Fig. 2 Receiver Operating Characteristic (ROC) curve for outpatient and 24-hour parameters, $T_{\max }$ and $\mathrm{IOP}_{\mathrm{var}}$, in detecting disease progression. As displayed, all plotted curves lie close to the reference line and expose the poor utility of these parameters for this purpose

\section{Table 1}

Table 1 $\#$ = number of; $\mathrm{R}$ ) = right eye; $\mathrm{L}$ ) = left eye; $\mathrm{CCT}=$ central corneal thickness; $\mathrm{MOPP}=$ mean ocular perfusion pressure

\begin{tabular}{|c|c|}
\hline Age (years) & $75.4 \pm 11.2$ \\
\hline $\begin{array}{c}\text { Female } \\
\text { Male }\end{array}$ & $\begin{array}{c}131(60 \%) \\
86(40 \%)\end{array}$ \\
\hline \# Drops & $\begin{array}{c}\text { R) } 2.3 \pm 1.4 \\
\text { L) } 2.3 \pm 1.4\end{array}$ \\
\hline \# Surgeries & R) $0.6 \pm 0.7$ \\
\hline L) $0.6 \pm 0.8$ \\
\hline MOP ( $\mu \mathrm{m})$ & R) $535.5 \pm 35.2$ \\
L) $533.4 \pm 37.7$ \\
\hline MmHg) & R) $59.2 \pm 9.0$ \\
\hline
\end{tabular}




\section{Table 2}

Table 2 Comparison of the demographic parameters of progressors and non-progressors in left eyes $\#$ = number of; $T_{\text {avg }}=$ average IOP; $T_{\max }=$ maximum IOP; IOP pressure

\begin{tabular}{|c|c|c|c|}
\hline Parameters & Progressors ( $\mathbf{n = 5 9 )}$ & Non-Progressors ( $\mathbf{n}=53)$ & $p$-value \\
\hline Age (years) & $72.6 \pm 11.1$ & $73.7 \pm 12.6$ & 0.46 \\
\hline $\begin{array}{c}\text { Female } \\
\text { Male }\end{array}$ & $\begin{array}{c}37(62.7 \%) \\
22(37.3 \%)\end{array}$ & $\begin{array}{l}27(50.9 \%) \\
26(49.1 \%)\end{array}$ & 0.25 \\
\hline \# Drops & $2.3 \pm 1.3$ & $2.5 \pm 1.5$ & 0.34 \\
\hline \# Surgeries & $0.7 \pm 0.8$ & $0.9 \pm 0.9$ & 0.49 \\
\hline $\mathbf{T}_{\text {avg }}$ (mmHg) & $13.7 \pm 2.5$ & $14.4 \pm 3.7$ & 0.87 \\
\hline $\mathbf{T}_{\text {max }}$ (mmHg) & $16.1 \pm 3.1$ & $16.9 \pm 4.4$ & 0.88 \\
\hline IOP $_{\text {var }}(\mathbf{m m H g})$ & $4.7 \pm 2.3$ & $5.0 \pm 2.5$ & 0.93 \\
\hline MOPP $(\mathbf{m m H g})^{6}$ & $61.0 \pm 8.7$ & $59.7 \pm 9.8$ & 0.92 \\
\hline
\end{tabular}

\section{Table 3}

Table 3 Utility of cut-off IOP values with sensitivity and specificity determined for cut-off points 15 and 22 $\mathrm{mmHg}$ for detecting glaucoma progression in left eyes

OP-IOP = IOP measurements during outpatient hours (10 AM, 2 PM, 5 PM)

\begin{tabular}{|c|c|c|c|c|}
\hline Cut-off value & parameter & 24h-IOP & OP-IOP & difference \\
\hline \multirow{2}{*}{$\mathbf{1 5} \mathbf{~} \mathbf{m H g}$} & sensitivity & $69.5 \%$ & $62.7 \%$ & $6.8 \%$ \\
& specificity & $26.4 \%$ & $35.8 \%$ & $-9.4 \%$ \\
\hline \multirow{2}{*}{$\mathbf{2 2} \mathbf{~ m m H g}$} & sensitivity & $3.4 \%$ & $1.7 \%$ & $1.7 \%$ \\
& specificity & $90.6 \%$ & $94.3 \%$ & $-3.7 \%$ \\
\hline
\end{tabular}




\section{Table 4}

Table 4 Bivariate correlations of various measured parameters between left and right eyes. All correlations had a p-value $<0.05$.

$\mathrm{T}_{\mathrm{avg}}=$ average IOP; IOP $=$ var $=$ IOP variation; $\mathrm{T}_{\max }=$ maximum IOP; $\mathrm{T}_{\min }=$ minimum IOP; \# = number of; RNFLT = Slope of Retinal Nerve Fiber Layer Thickness decrease; $\mathrm{G}=$ Global; $\mathrm{TS}=$ temporal-superior; $\mathrm{T}=$ temporal; $\mathrm{TI}=$ temporal-inferior

\begin{tabular}{|c|c|c|}
\hline Parameter & r-Value & Strength of Correlation \\
\hline$T_{\text {avg }}$ & 0.51 & moderate \\
\hline $10 P_{\text {var }}$ & 0.65 & strong \\
\hline $\mathbf{T}_{\max }$ & 0.78 & strong \\
\hline $\mathbf{T}_{\text {min }}$ & 0.48 & moderate \\
\hline \# drops & 0.76 & strong \\
\hline \# surgeries & 0.48 & moderate \\
\hline $\begin{array}{c}\text { RNFLT loss } \\
\text { G } \\
\text { TS } \\
\text { T } \\
\text { TI }\end{array}$ & $\begin{array}{l}0.43 \\
0.33 \\
0.29 \\
0.32\end{array}$ & $\begin{array}{c}\text { moderate } \\
\text { weak } \\
\text { weak } \\
\text { weak }\end{array}$ \\
\hline MOPP & 0.96 & very strong \\
\hline
\end{tabular}




\section{$\underline{\text { Figures }}$}

\section{Figure 1}

Figure 1. Average IOP values measured at the 5 nycthemeral monitoring protocol times. The peak IOP was recorded at 10

am and

the trough

at

pm.

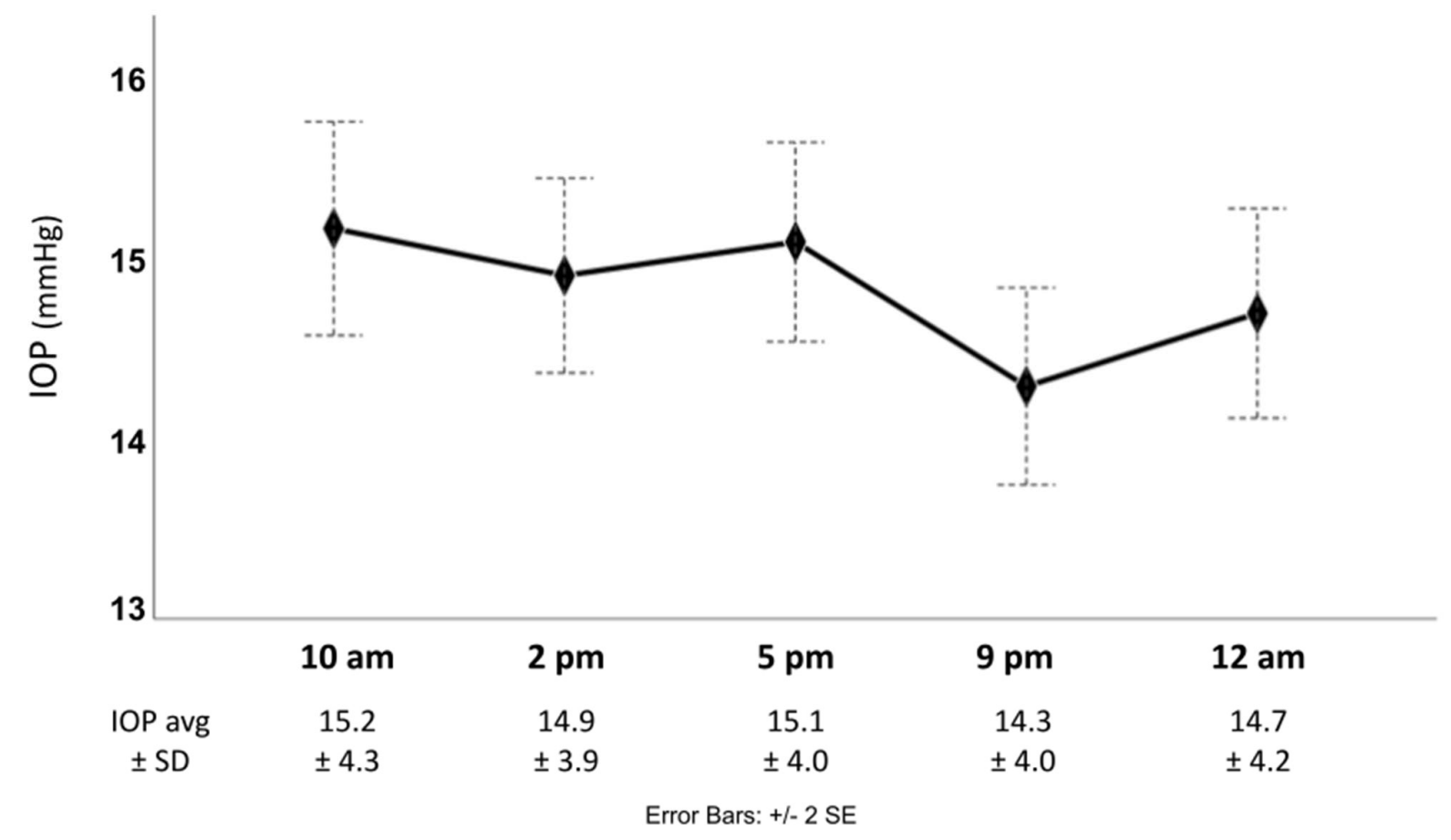




\section{Figure 2}

Figure 2: Receiver Operating Characteristic (ROC) curve for outpatient and 24-hour parameters, $T_{\max }$ and IOP $_{\text {var }}$ in detecting disease progression. As displayed, all plotted curves lie close to the reference line and expose the poor utility of these parameters for this purpose.

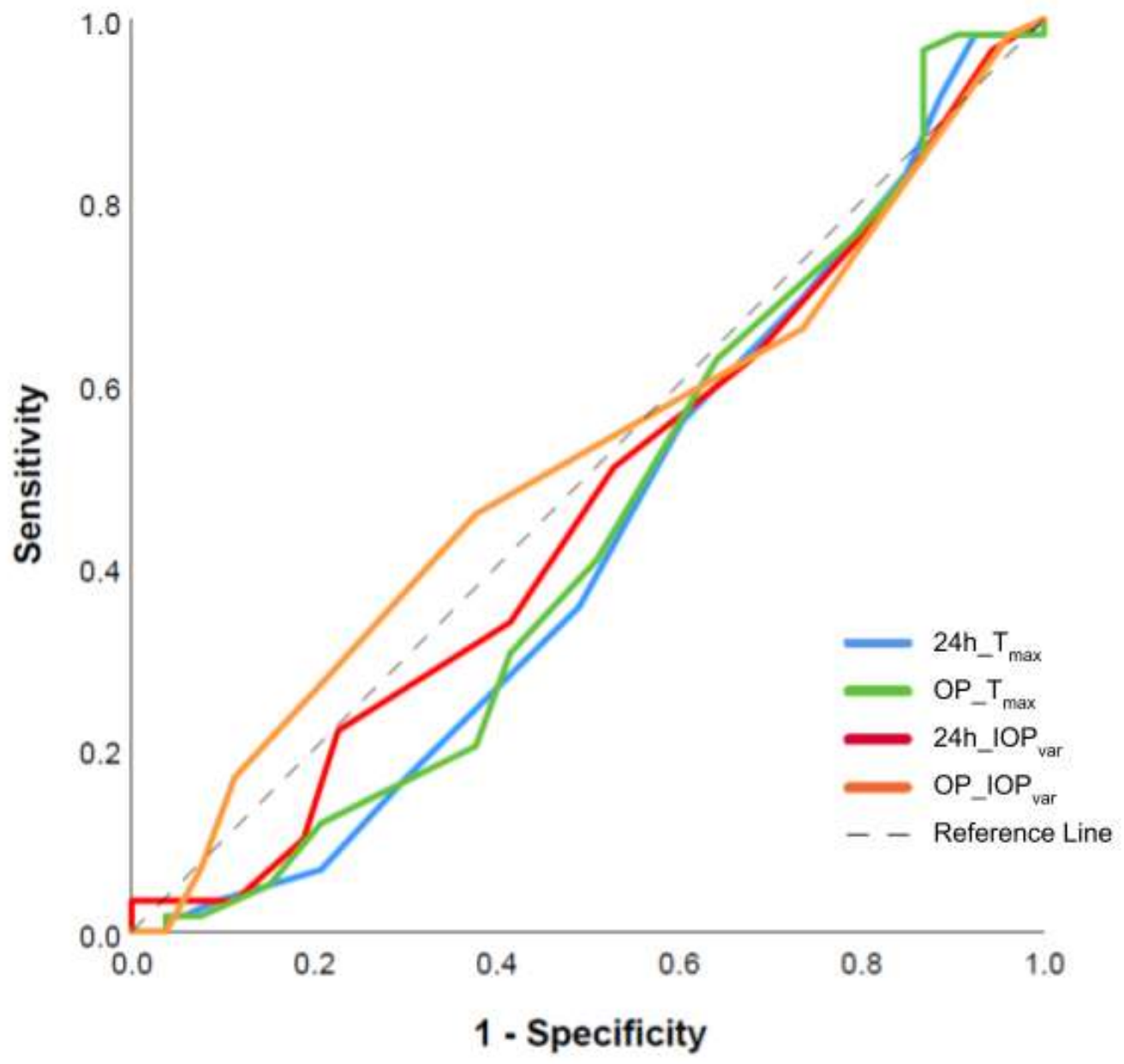

